\title{
Reducing latent errors, drift errors, and stakeholder dissonance
}

\author{
George M. Samaras \\ Samaras \& Associates, Inc., 7755 Soda Creek Road, Pueblo, CO 81005, US
}

\begin{abstract}
Healthcare information technology (HIT) is being offered as a transformer of modern healthcare delivery systems. Some believe that it has the potential to improve patient safety, increase the effectiveness of healthcare delivery, and generate significant cost savings. In other industrial sectors, information technology has dramatically influenced quality and profitability - sometimes for the better and sometimes not. Quality improvement efforts in healthcare delivery have not yet produced the dramatic results obtained in other industrial sectors. This may be that previously successful quality improvement experts do not possess the requisite domain knowledge (clinical experience and expertise). It also appears related to a continuing misconception regarding the origins and meaning of work errors in healthcare delivery. The focus here is on system use errors rather than individual user errors. System use errors originate in both the development and the deployment of technology. Not recognizing stakeholders and their conflicting needs, wants, and desires (NWDs) may lead to stakeholder dissonance. Mistakes translating stakeholder NWDs into development or deployment requirements may lead to latent errors. Mistakes translating requirements into specifications may lead to drift errors. At the sharp end, workers encounter system use errors or, recognizing the risk, expend extensive and unanticipated resources to avoid them.
\end{abstract}

Keywords: human factors, healthcare, technology, quality, improvement

\section{Introduction}

Healthcare information technology (HIT) is being offered in the United States (US) as an important element in the transformation of modern healthcare delivery systems $[5,28,15]$. Relatively low implementation success rates and misguided beliefs of its role calls into question HIT's ultimate impact on quality and cost-effectiveness $[23,4]$. The healthcare delivery business tends to be revenue constrained. Most revenues are from private and public third-party payers and are relatively inelastic. Capital equipment and consumable supply costs are inflated due to various market factors, including US federal regulation. As a result, increasing revenues cannot solve existing problems and extracting cost-savings from existing processes through quality improvement appears to be the best way forward. Quality assessment and improvement in healthcare has been an active endeavor in the US at least since 1992 [17].
The sole purpose for development and deployment of any product, process, or service is an attempt to meet some or all the stakeholders' needs, wants, and desires (NWDs). A stakeholder is a well-established management concept [25] and refers to any individual, or group of individuals, who have an ability to affect the outcome of an endeavor in a positive or negative manner. Stakeholders may be characterized by their evolving and often conflicting NWDs (Need - a basic need or "I must have it", Want - a performance need or "I would like to have it", and Desire a latent need or "I'll know it when I see it"). Stakeholder dissonance describes conflicting NWDs that result in errors, workarounds, and threats to patient safety and organizational profitability [8]. Conflicting NWDs often arise following changes to organizational work processes very often associated with the introduction of new technologies.

Job redesign [1] is a key tool in quality improvement and has been studied and applied across the 
whole spectrum of workers, including physicians [16, 20]. Job redesign affects the character of work, including workflow, workload, and worker satisfaction; it may be intentional or inadvertent. "Changes in the character of work following introduction of HIT are associated with errors, workarounds and threats to patient safety implying that it may be creating stakeholder dissonance [8].

\section{Quality and improvement}

The quality movement traces its roots back to the $13^{\text {th }}$ century, when craftsmen began organizing in guilds [3]. These guilds developed strict rules for product and service quality and inspection committees enforced the rules with a special mark; the craftsmen added a second mark, which ultimately became a symbol of the craftsman's good reputation ... for good quality. Following the Industrial Revolution, the craftsmen's trades were divided into specialized tasks. Later, these tasks were further subdivided into simple operations and the craftsmen were essentially eliminated in many industrial sectors. While this dramatically increased productivity, it often had the opposite effect on quality. This led to the use of inspectors, who would prevent poor quality products from reaching customers. Inspection alone proved inadequate and in the $20^{\text {th }}$ century quality management moved successively through statistical quality control, quality assurance, and strategic quality management. This was the process approach to quality practices, a process being defined as a group of activities that accepts an input, adds some value, and produces an output. This was followed by business process reengineering, whose objective was rapidly to redesign strategic processes to optimize the workflow and productivity of an organization. At the same time, a wide variety of continuous quality improvement techniques were developed and proved successful in non-healthcare industrial sectors. They included two complimentary techniques: "lean" [19] whose objective is to optimize the adding of value by eliminating waste in the serial and parallel sequences of related processes (the value stream) and "six sigma" [27] whose objective is to minimize process variability.

Outside the healthcare delivery domain, where quality is more readily defined in individual industrial sectors for individual products and services, quali-

*Corresponding author. E-mail: george@samaras-assoc.com ty improvement often had dramatic beneficial effects. Quality improvement practitioners in these domains often developed a high degree of expertise and impressive track records - but these seemed not to translate well into the healthcare delivery domain. In part, this may be due to the quality improvement experts' lacking the requisite domain (craft) knowledge. Figure 1 illustrates this, showing the quality improvement solution space as a function of both domain knowledge and quality improvement knowledge. But this cannot be the complete explanation. In part, it also may be attributable to difficulties arising from the inherent tension between the search for high throughput and the involvement of primarily professional workers (craftsmen). But neither can this be the complete explanation. We also must consider how the quality objective is defined in the healthcare delivery domain.

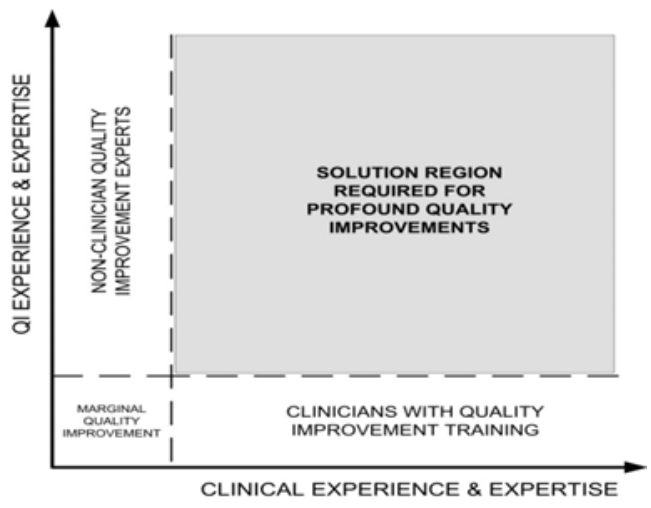

Fig. 1 Quality improvement solution space illustrating the region requiring combined clinical and engineering expertise.

There exist a myriad "definitions of quality" and many believe that the concept of quality is elusive. However, a human-centered definition of quality subsumes all others I have examined; it is: quality is the degree to which the needs, wants, and desires of all the stakeholders have been satisficed $[8,11]$. The term satisfice is presumed to be a contraction of the terms satisfy and suffice coined by Simon [14], who defined it as obtaining a good result that is good enough, though not necessarily the best, for each stakeholder. Berwick [7] identifies six dimensions of healthcare performance: safety, timeliness, effectiveness, efficiency, equity, and patient-centeredness. These are not independent dimensions of quality [11] and are thus inappropriate for quality improvement efforts. Using interdependent dimensions of quality 
with an implicit assumption that they are independent may undermine the effectiveness of both the analyses and the subsequent interventions.

Four quality dimensions that are independent and

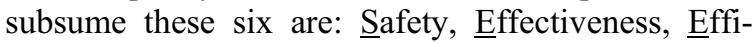
ciency, and Satisfaction (SEES) $[12,11]$. These correspond to the top-level NWDs for all stakeholders [12] - though different stakeholders may define them differently. The first three are objective measures (safety, effectiveness, and efficiency). The fourth (satisfaction) is a set of five subjective measures (perceived effectiveness, perceived efficiency, engaging, error tolerant and easy to learn) [11].

Safety is not just a matter of avoiding physical, psychological, or socioeconomic injuries to patients, but also avoiding such injuries to other stakeholders, including clinicians, support staff, and healthcare delivery organizations. Effectiveness is not only provision of evidence-based "treatment", but also provision of that treatment to all where (location) and when (timeliness) they may benefit; "treatment" needs to be understood in the broadest sense for all stakeholders (not just receiving a pill, but also having your work structure changed, your reimbursement terms altered, etc.). Efficiency is about avoiding wasting resources, but is totally dependent upon where you draw your system boundary (your frame of reference), since only what crosses the system boundary may be included in an efficiency calculation [24]. Timeliness is not an orthogonal quality dimension; it is an element of effectiveness (providing treatment at a time it will be beneficial), efficiency (not wasting time), and satisfaction (because, as previously stated, satisfaction is a function of perceived effectiveness and perceived efficiency). Patient-centeredness (while the raison d'être of healthcare delivery) is not an independent dimension of quality; it is but one of a number of foci of the complete set of stakeholders that must be balanced in the implementation of a rational healthcare delivery system. Finally, equity (providing care invariant over demographic and socioeconomic status) does not survive careful analysis as an independent quality dimension (even though it is very attractive from a social justice perspective). Inequitable distribution of care jeopardizes the safety of some patients, is ineffective from a public health perspective (think of herd immunity), is inefficient from a national economic perspective (think of who is paying for whom to go to the emergency room), and is dissatisfying to many of the stakeholders (not all of whom are merely the recipients of inequitable care) [11].

\section{Complexity and errors}

Introducing human actors (a term that subsumes end-users) into any endeavor dramatically increases the possible number of incorrect or inappropriate responses of a "simple" hardware/software system. Human actors, and their organizations, drastically increase system complexity. Introduction of HIT in healthcare delivery is an example of essential complexity; it is unrealistic to believe that "simple" solutions will adequately address the requisite solution space (Figure 1). Unfortunately, this compounds the already nonlinear, and often stochastic, coupling among healthcare delivery system components, leading to what may be characterized as a hypercomplex system - a polycentric system with complexity (the HIT) inscribed within complexity (the existing healthcare delivery system) [21].

Complex systems have emergent properties; they are the result of component interactions at the interfaces that are not readily predictable without appreciation of the system as a whole. Emergent properties may be useful, benign, annoying, or dangerous; in all instances, they arise at system interfaces, especially the human-system interfaces. Not fully appreciating human-centered system complexity has been an important obstacle in the development and deployment of essential systems. We now recognize that development-induced and deployment-induced errors are a serious problem, may become critical safety issues, and are an important source of reduced quality. They are rarely eliminated merely with labeling or user training!

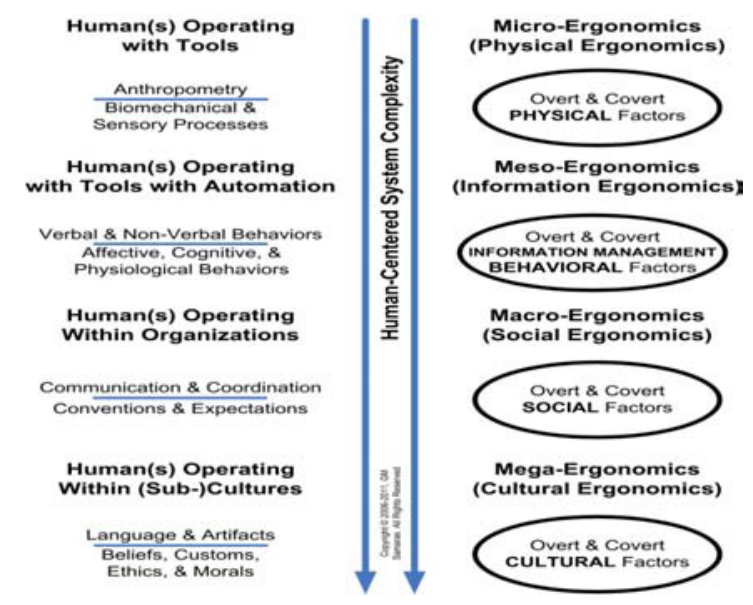

Fig. 2 Human-centered system complexity model illustrating four levels of human-system interfaces. Adapted from [12]. 
Figure 2 models the range of interfaces from individuals and their tools to groups of individuals operating towards putatively common goals. There are both overt and covert aspects to these human-system interfaces. It is these overt and covert human interfaces that are the basis for stakeholder NWDs in any system. Unmitigated conflicting NWDs among system stakeholders lead to stakeholder dissonance and often result in the opportunities for system use errors.

Errors are epiphenomena of complex system interfaces. They are symptoms, not root causes. Figure 3 shows a taxonomy of such errors organized by error category versus error type. In the category of system use errors, Reason [18] has distinguished active errors (the result of known development/deployment "bugs") and latent errors (the result of unknown development/deployment "bugs"). Dekker [26] identifies drift errors - a misguided, usually slow, incremental progression of systems operations taking the tool beyond the designed safety envelope. Finally, we have malicious corruption of the tool by non-endusers (sabotage). Human errors in the system use and individual user categories are not mutually exclusive; very often failures occur when multiple contributors err - each necessary, but only jointly sufficient [6], resulting in failure.

\begin{tabular}{|c|c|c|}
\cline { 2 - 3 } \multicolumn{1}{c|}{} & \multicolumn{2}{c|}{ ERROR CATEGORY } \\
\hline ERROR TYPE & $\begin{array}{c}\text { SYSTEM USE } \\
\text { ERROR }\end{array}$ & $\begin{array}{c}\text { INDIVIDUAL USER } \\
\text { ERROR }\end{array}$ \\
\hline EXPECTED BEHAVIOR & $\begin{array}{c}\text { ACTIVE } \\
\text { (KNOWN BUGS) }\end{array}$ & ROUTINE USE \\
\hline UNEXPECTED Behavior & $\begin{array}{c}\text { LATENT } \\
\text { (UNKNOWN BUGS) }\end{array}$ & NOVEL USE \\
\hline MISGUIDED Behavior & $\begin{array}{c}\text { DRIFT } \\
\text { (BEYOND DESIGN ENVELOPE) }\end{array}$ & MISUSE \\
\hline MALICIOUS Behavior & SABOTAGE & ABUSE \\
\hline \multirow{2}{*}{$\begin{array}{c}\text { LOCUS OF CONTROL: } \\
\text { DEVELOPMENT ORGANZATION } \\
\text { \& DEPLOMMENT ORGANIZATION }\end{array}$} & $\begin{array}{c}\text { LOCUS OF CONTROL: } \\
\text { INDIVIDUAL } \\
\text { HUMAN(S) }\end{array}$ \\
\hline
\end{tabular}

Fig. 3 Human-centered error taxonomy illustrating the difference between a system use error and an individual user error.

Our focus here is on system use errors, not individual user errors. Errors associated with malicious behaviors are also not considered. While all errors appear only when the system is being used by the individual user, system use errors may be viewed as properties of the system, whereas individual user errors must be viewed as properties of the individual. The source of system use error opportunities for endusers is the developing and deploying organizations, not the end-users. This is an important distinction; the HIT manufacturer is not the only source of system use errors; the HIT deployer may compound development-induced system use errors or introduce new system use errors.

\section{Development \& deployment processes}

In order for HIT to be introduced into the healthcare delivery system, first it must be developed by a manufacturing organization and then it must be deployed by a user organization. Figure 4 graphically describes origins of active, latent, and drift system use errors during HIT development. Inadequate identification of all the stakeholders or their NWDs invariably leads to ignorance of conflicting stakeholder NWDs. The system development requirements (design inputs) are a subset of the stakeholder NWDs chosen because they are technologically and economically feasible at the beginning of the development effort. Missing or misunderstanding stakeholder NWDs may result in flawed design inputs. Missing or misunderstanding design inputs may result in flawed system specifications (design outputs which are the engineers' work product).

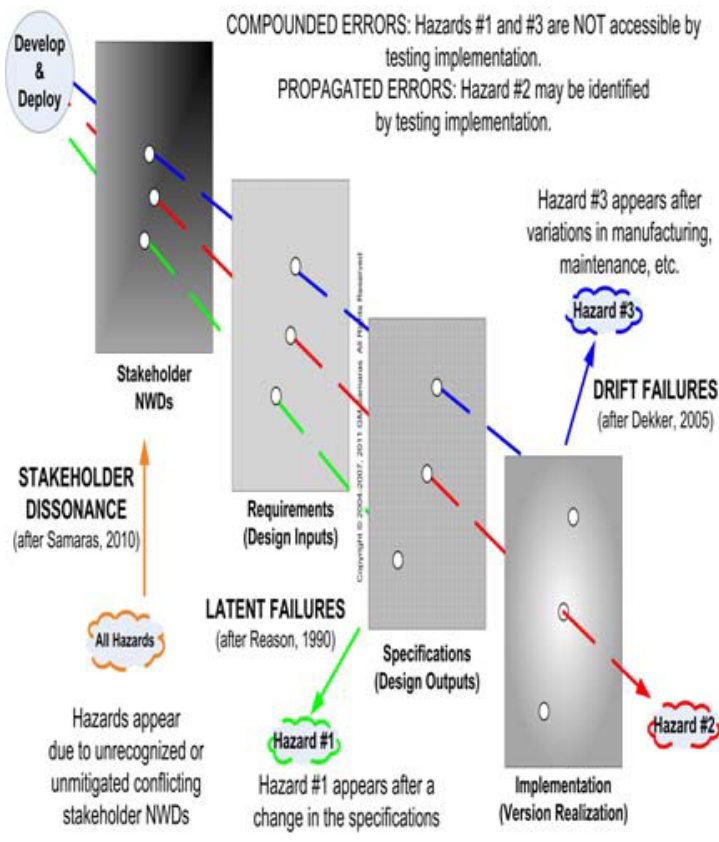

Fig. 4 Generation of system use errors: active (Hazard \#2), latent (Hazard \#1) and drift (Hazard \#3). Adapted from [10, 13]. 
Flawed design inputs can result in latent "bugs", because engineers do what they know works regardless of the documented requirements (the green line is blocked by the use of traditional engineering specifications). Well-intentioned subsequent changes in system specifications permit the flaw, no longer latent, to appear in the physical implementation of the system. Missing or misunderstood design outputs may set the stage for drift into failure. Wellintentioned subsequent variations in manufacturing or maintenance may push the system beyond its designed safety envelop allowing a no-longer hidden hazard to appear.

Figure 4 could also be constructed to show deployment-induced active, latent, and drift system use errors. The stakeholders now would be identified with far more specificity. Their NWDs would be the basis for the deployment requirements. The deployment specifications would be documented as configurations, standard operating procedures (SOPs), work instructions (WIs), and any associated training materials, while the implementation is what is colloquially called "going live". The new SOPs/WIs impact job redesign, workflow, and workload; they may improve quality or diminish it.
Figure 4 also shows the critical limitation of system validation [10], which is the empirical demonstration that the system as implemented conforms to the design inputs. System validation is the only defense against errors of the third kind - "correctly solving the wrong problem" [9]. If a hazard does not exist as a system property when the validation is conducted, it cannot possibly be detected. Since validation is based solely on testing the implemented system against the documented design inputs, if a design input is absent (a latent flaw), the tool will incorrectly pass validation testing. In the case of periodic revalidations after deployment (routinely required in pharmaceutical manufacturing), maintenance requirements that were not anticipated (another latent flaw) or that deviate from the original design (a drift towards failure) may also result in the tool incorrectly passing re-validation.

Considering the development and deployment processes independently obscures the critical linkages between these lifecycle processes and how development and deployment system use errors may be propagated or mitigated.

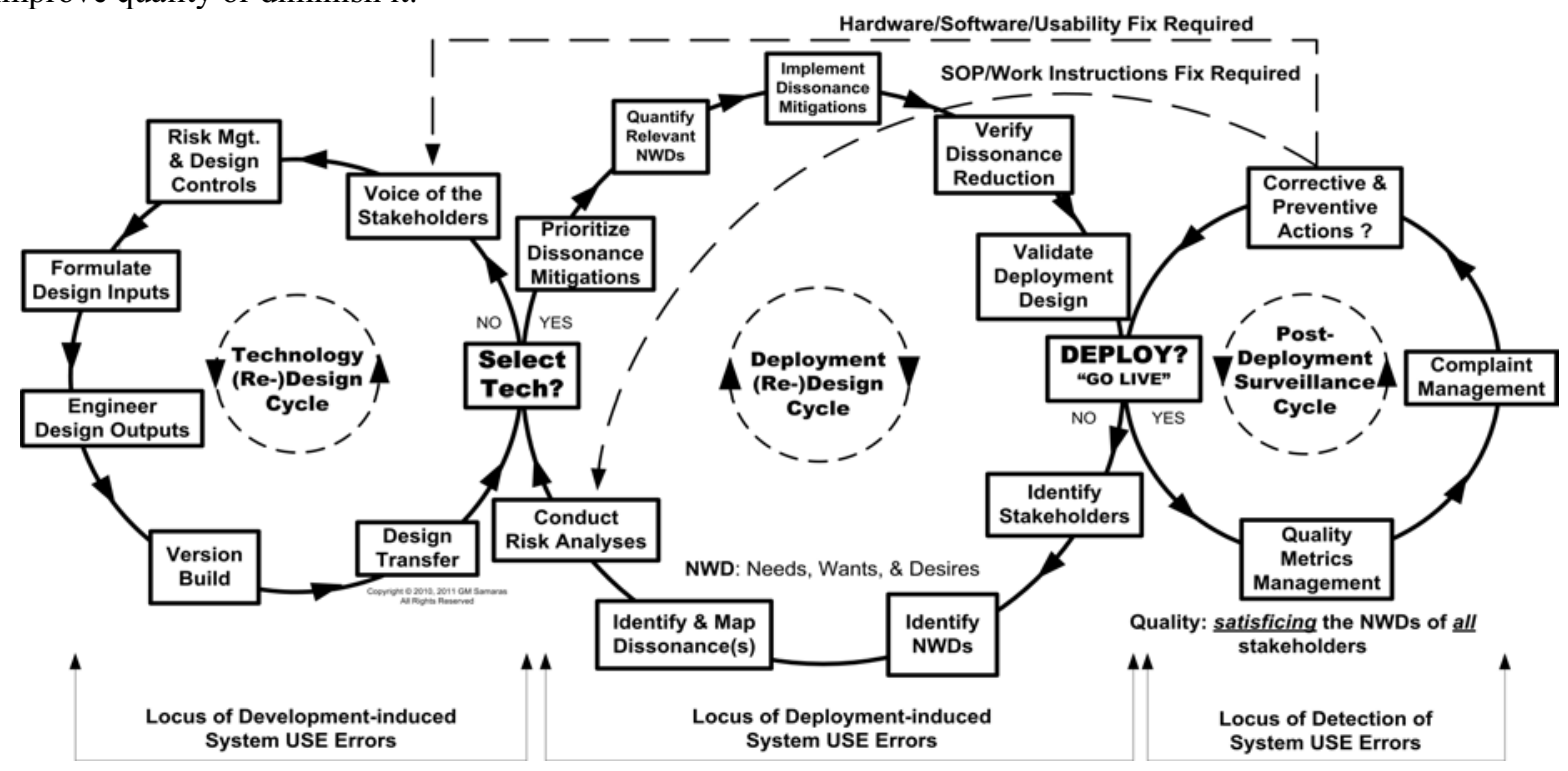

Fig. 5 Linkages in development, deployment, and post-deployment lifecycles. Adapted from [11].

\section{Defense against system use errors}

Development and deployment lifecycles are linked (Figure 5) permitting system design flaws to be prop- agated or even compounded from development through deployment to the individual users, who experience these system properties as system use errors. These lifecycles are iterative processes. Their inherent linkage permits reduction or expansion of system 
use error opportunities. For example, extension by the developer of concurrent engineering practices to include representatives of the deploying organizations during initial development (not after the system has been sold and is being "fixed") offers a path to reduced development-induced system use errors or recognition of these errors during deployment design.

The deploying organization may also minimize deployment-induced system use errors by focusing on detailed stakeholder identification, soliciting stakeholder SEES perspectives, and translating these to NWDs. This permits identifying NWD conflicts, which may then be prioritized for mitigation based upon risk. Stakeholder dissonance mitigations in the chosen configurations and SOPs/WIs need to be verified and the deployment design must be validated prior to "going live". Typically, validation occurs in a restricted, but representative, setting. As with product design validation, deployment design validation is far more cost-effective before deploying the system to the users. The cost of fixing deploymentinduced system use errors, as well as propagated development-induced system use errors, is well-known to be orders of magnitude more costly after the system has "gone live".

Reducing stakeholder dissonance, latent and drift system use errors is not the same as completely eliminating them. A third lifecycle process (Figure 5) needs to be linked to the development and deployment lifecycle processes. A post-deployment surveillance process characterized by systematic collection of quantitative quality metrics and comprehensive complaint management can lead to early (and far less costly) corrective and preventive actions that may feedback to both the deployment and development designs.

\section{A familiar example}

Nurses and pharmacists are quite familiar with prescriber orders being found in a comment field of a computerized provider order entry system. One might be tempted to claim that this is an intentional individual user error, not a system use error; if that were the case, it would be malicious behavior by the prescriber (Figure 3). This might be the result of time pressure or affective behaviors motivated by anger or frustration causing the prescriber to take temporarily a path of least resistance. There is a greater likelihood that the prescriber is not behaving maliciously and that this is the expression of a system use error.

Figure 6 shows a partial root cause analysis using an Ishikawa diagram, which is one of the seven basic tools of quality [2]. This is a deductive analysis of the order entry event. Active system use errors that could be responsible include usability issues such as difficulty navigating to the desired screen or the particular drug is not available in the hospital's customized drug library. Latent system use errors that could be responsible include a software defect on the particular screen or defective user training and support procedures in the hospital. Drift system use errors might include a flawed software upgrade that eliminated the custom drug library (so the drug could not be selected from the drop-down list) and/or failure of the rapid response team to properly re-validate the system after the software upgrade and before redeployment.

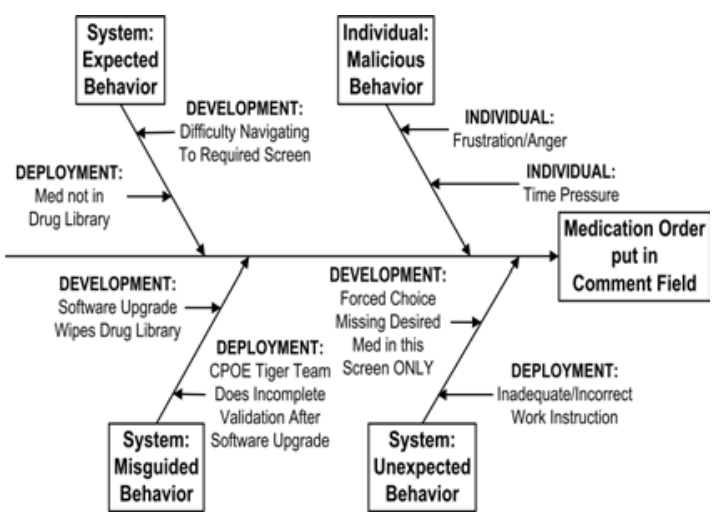

Fig. 6 Partial root cause analysis of undesirable order entry event.

So, while it is simple to blame the prescriber who, after all, was clearly the one that actually "did it", this conclusion is logically flawed; end-user sanctions, counseling, retraining, or job redesign will NOT address system use errors.

\section{Conclusion}

Quality improvement requires both a clear understanding of quality's independent dimensions in the healthcare delivery domain and how to diagnose and treat errors that adversely impact quality. Four measureable dimensions of quality (SEES) have been discussed; three are objective measures and the fourth requires a set of subjective measurements. 
Soliciting SEES perspectives from all the stakeholders permit identification of their NWDs and potential conflicts [11]. Well-characterized stakeholder NWDs support the formulation of complete and correct design inputs. Properly operationalized design inputs support the engineering of complete and correct design outputs; they also provide the basis for complete and correct system validations.

Minimizing human error first requires that we recognize which humans err. This requires that we discriminate between system use errors and individual user errors; each may be characterized by the same four types of behaviors (Figure 3). The focus here has been on system use errors, not individual user errors.

System use errors are generated by the developers and deployers of the HIT system. Reducing latent errors and drift errors in HIT initially requires identification and reduction of stakeholder dissonance. Fundamentally, this is a requirement that both the developer and the deployer fully recognize and understand ALL the stakeholders (both as individuals and as organizations), their work and work environment, and the human interfaces to the HIT.

Development-induced system use errors may be reduced by extending concurrent engineering practices to include deployer representatives - an interdisciplinary participative cooperative approach [22] often called Team Science [23]. Deploymentinduced system use errors may be reduced by formalizing the deployment design process that focuses on job design, workflow, workload, and work quality (SEES) - realizing that the objective should be minimum excursions from already working processes and procedures.

If healthcare delivery quality improvement is to match the great successes in other industrial sectors, the quality improvement experts must have the requisite domain knowledge (clinical experience and expertise). While interdisciplinary teamwork is important, diagnosing and treating quality problems in healthcare delivery appears to demand intuitive understanding of clinical practice. There is certainly evidence of clinicians seeking engineering or quality improvement training; there is also evidence of quality improvement professionals seeking clinical knowledge. For the most part (there are notable exceptions), this seems to have been unsatisfactory, in that the clinicians really never attain the requisite engineering skill set and the engineers really never inculcate the clinical mindset. As with every other societal problem, there are short-term solutions (utilizing only independent, domain relevant dimensions of quality in quality improvement research and practice), medium-term solutions (modifying HIT development processes and HIT deployment processes to focus on all the stakeholders and stakeholder dissonance), and long-term solutions (interdisciplinary training of engineer-clinicians).

\section{References}

[1] A. M. Grant, Y. Fried, T. Juillerat, Work Matters: Job Design in Classic and Contemporary Perspectives. in S. Zedeck ed., APA Handbook of Industrial and Organizational Psychology, 1 ( 2010): 417-453. Washington, DC: American Psychological Association.

[2] American Society for Quality. The Basic Tools of Quality. http://asq.org/learn-about-quality/seven-basic-qualitytools/overview/overview.html (accessed August 29, 2011)

[3] American Society for Quality. The History of Quality Overview. http://asq.org/learn-about-quality/history-ofquality/overview/overview.html (access August 29, 2011)

[4] B-T. Karsh, M.B. Weinger, P.A. Abbott and R.L. Wears, Health information technology: fallacies and sober realities, J. Am. Med. Inform. Assoc. 17 (2010):617-623.

[5] D. Blumenthal, Launching HITECH. N. Engl. J. Med. 362 (2010):382-5

[6] D.D. Woods and E. Hollnagel, Prologue: Resilience Engineering Concepts in Resilience Engineering, Hollnagel, Woods, \& Leveson, eds, Hampshire, England: Ashgate, 2006, pg 3 .

[7] D.M. Berwick, A user's manual for the IOM's "quality chasm" report. Health Affairs, 21(3) (2002):80-90.

[8] E.A. Samaras and G.M. Samaras, Using Human-Centered Systems Engineering to Reduce Nurse Stakeholder Dissonance. Biomedical Instrumentation \& Technology 44(s1) (2010):25-32

[9] F. Mosteller, A k-sample slippage test for an extreme population, Ann Math Stat. 19(1) (1948): 58-65

[10] G M. Samaras, An Approach to Human Factors Validation. J. Validation Technology 12(3) (2006):190-201

[11] G.M. Samaras, Human-Centered Systems Engineering: Managing Dissonance in Healthcare Delivery, in Management Engineering for Effective Healthcare Delivery: Principles and Practices, A. Kolker and P. Story, eds., Philadelphia: IGI Global 2011, pg. 148-171.

[12] G.M. Samaras and E.A. Samaras, Feasibility of an e-health initiative: Information NWDs of cancer survivor stakeholders. Proceedings of IEA 2009. Beijing, China. August 9-14, 2009.

[13] G.M. Samaras, The use, misuse, and abuse of design controls. IEEE Engineering in Medicine and Biology Magazine, 29(3) (2010):12-18

[14] H.A. Simon, Models of man: Social and rational. New York, NY: Wiley, 1957.

[15] Healthcare Information and Management Systems Society. Transforming Healthcare through IT. http:/www.himss.org (accessed August 29, 2011).

[16] J. Hoffer Gittell, D.B. Weinberg, A.L. Bennett, and J.A. Miller, Is The Doctor In? A Relational Approach to Job Design and the Coordination of Work. Human Resource Management 47(4) (2008): 729-755.

[17] J. M. Luce, A.B. Bindman, and P.R. Lee, A Brief History of Health Care Quality Assessment and Improvement in the United States. West J. Med. 160 (1994): 263-268. 
[18] J. Reason, Human Error, Cambridge: Cambridge University Press, 1990

[19] J.P. Womack, D.T. Jones, and D. Roos, The machine that changed the world: The story of lean production -- Toyota's secret weapon in the global car wars that is now revolutionizing world industry. New York, NY: Free Press, 1990.

[20] K.M. Unertl, L.L. Novak, K.B. Johnson, and N.M. Lorenzi, Traversing the many paths of workflow research: developing a conceptual framework of workflow terminology through a systematic literature review, J. Am. Med. Inform Assoc. 17 (2010):265-273.

[21] L. Qvortrup, The Hypercomplex Society. New York: Peter Lang, 2003.

[22] M. Dixon-Woods, R. Amalberti, S. Goodman, B. Bergman, and P. Glasziou, Problems and promises of innovation: why healthcare needs to rethink its love/hate relationship with the new, BMJ Qual. Saf. 20(s1) (2011):i47-I51

[23] N.M. Lorenzi, L.L. Novak, J.B. Weiss, C.S.Gadd, and K.M. Unertl, Crossing the Implementation Chasm: A Proposal for
Bold Action. J. Am. Med. Inform. Assoc. 15 (2008): 290296.

[24] R. Dubin, Organizational effectiveness: Some dilemmas of perspective. In S. Lee (Ed.), Organizational effectiveness: Theory-research-utilization, (p. 7). Kent, OH: Kent State University Press. 1976.

[25] R. Phillips, R.E. Freeman, and A.C. Wicks, What Stakeholder Theory Is Not. Business Ethics Quarterly 13(3) (2003):479-502.

[26] S.W.A. Dekker, Ten Questions about Human Error: A New View of Human Factors and System Safety, New Jersey: Lawrence Erlbaum Associates, 2005.

[27] T. Pyzdek, Quality Engineering Handbook. Tucson, AZ: Quality Publishing, 2003.

[28] The Leapfrog Group. The Leapfrog Groups for Patient Safety. http://www.leapfroggroup.org/home (accessed August 29, 2011) 